\title{
LOS GUISANTES MÁGICOS DE DARWIN Y MENDEL
}

\author{
Andrés Galera \\ Depto. $\mathrm{H}^{\mathrm{a}}$ Ciencia, Instituto de Historia, CSIC.
}

\section{RESUMEN}

En el presente trabajo analizamos los modelos hereditarios propuestos por Darwin y Mendel para explicar la historia evolutiva de la Tierra. De la comparación resultan dos teorías biológicas enfrentadas, regidas una por el principio de la selección natural y la otra por la mutación.

\section{SUMMARY}

Presently work analyzes the hereditary models proposed by Darwin and Mendel to explain the evolutionary history of the Earth. The comparison shows us two faced biological theories, governed, one, for the principle of the natural selection, the other for the mutation.

Desde su publicación en 1866 las leyes formuladas por Mendel ${ }^{1}$ sobre la herencia de caracteres en la reproducción sexual tuvieron desigual fortuna científica. Olvidadas durante más de treinta años, tras su redescubrimiento en 1900 han sido objeto de culto mundial ${ }^{2}$. Contemporáneamente, la hipótesis evolucionista desarrollada por Darwin en su libro On the origin of species by means of natural selection suscitó entusiasmo y controversia desde 1859 hasta hoy. La relación historiográfica trazada alrededor de ambos científicos presenta también cierta discriminación, pues el rango alcanzado por Darwin como patriarca del evolucionismo moderno se conjuga mal con un modelo de herencia mendeliano que redefine a la baja el papel desempeñado por la selección natural darwinista en la evolución de los organismos. La pregunta es obligada y no requiere mayores preámbulos, ¿conoció Darwin las leyes de Mendel?

1 Mendel, G. (1866) «Versuche über Pflanzen-hybriden», Verhandlungen des Naturforschenden Vereins, Brünn, IV, pp. 3-47. Para analizar las leyes mendelianas utilizamos la siguiente edición italiana: Mendel, G. (1984), Le leggi dell'ereditarietà, Milán, Rizzoli.

2 En los primeros siete años de la centuria el artículo de Mendel se tradujo al inglés: Journal of the Royal Horticultural Society, 26, 1901, pp. 1-32; y al francés: Bulletin Scientifique de la France et de la Belgique, 41, 1907, pp. 371-419. 
Generalmente, los historiadores de la biología resolvemos este problema manteniendo a los personajes en áreas separadas: genética y teoría de la evolución, convirtiéndoles en modernos biólogos fundadores de las respectivas disciplinas. La interpretación es anacrónica y engañosa, es una argucia para evitar confrontar sus ideologías. Durante mucho tiempo el principal argumento esgrimido a favor del supuesto aislamiento fue la escasa difusión del artículo de Mendel. Hace años que se demostró la falsedad de la hipótesis. Separatas y ejemplares de la revista editada por la Sociedad de Ciencias Naturales de Brünn conteniendo el artículo de Mendel se localizaban antes de 1900 en las bibliotecas de relevantes instituciones científicas. La Royal Society, la Linnaean Society, la Library of Congress and Smithsonian Institution, la Library of the Museum of Comparative Zoology de la Universidad de Harvard, la Académie Royale des Sciences Naturelles de Bruselas, la Société des Sciences Naturelles de Estrasburgo, son un pequeño ejemplo de su difusión por Europa y Norteamérica a finales de $\operatorname{siglo}^{3}$. Durante la década de los años setenta las leyes mendelianas traspasaron el umbral universitario, debatiéndose públicamente en las universidades de Upsala y San Petersburgo en 1872 y 1874 respectivamente ${ }^{4}$. Los hechos son concluyentes, antes de 1900 el artículo redactado por Mendel exponiendo su teoría hereditaria estaba al alcance de la comunidad científica internacional. ¿Podemos establecer un vínculo directo entre Darwin y Mendel? La pregunta tiene respuesta a través del libro de Hermann Hoffmann Untersuchungen zur Bestimmung des Werthes von Species und Varietät ${ }^{5}$, publicado en 1869, donde se recogen los experimentos mendelianos ${ }^{6}$. En 1876 se editó el libro de Darwin The effects of cross and self fertilisation in the vegetable kingdom, su lectura revela que Darwin leyó y analizó el libro de Hoffmann y las referencias conducen a las páginas que contienen la información relativa a Mendel ${ }^{7}$. Consecuentemente, entre 1869 y 1876 Darwin tuvo la posibilidad de conocer la teoría hereditaria expuesta por el monje pero, intencionadamente o no,

3 Cf. FISHER, R.A. (1936), «Has Mendel Work Been Rediscovered?», Annals of Science, vol. 1, $\mathrm{n}^{\circ}$ 2, pp. 134-137; OlBY, R. \& GAUTREY, P. (1968), «Eleven References to Mendel Before 1900», Annals of Sciences, vol. 24, pp. 7-20; Olby, R. (1985), Origins of Mendelism, New York, Schocken, $2^{\mathrm{a}}$ ed. rev., pp. 216-234 (1 $1^{\text {a }}$ ed., New York, Schocken, 1966); WEIR, J. A. (1968), «Agassiz, Mendel, and Heredity», Journal of the History of Biology, vol. 1, pp. 179-293; WEINSTEIN, A. (1977), «How Unknown Was Mendel's Paper?», Journal of the History of Biology, vol. 10, pp. 341-364; SERMONTI, G. (1984), Mendel, nascita e rinascita della genetica, Brescia, La Scuola.

4 Cf. WEINSTEIN, A. (1977), pp. 343-344.

5 Hoffmann, H. (1869), Untersuchungen zur Bestimmung des Werthes von Species und Varietät. Ein Beitrag zur Kritik der Darwin'schen Hypothese, Giessen, J. Ricker'sche Buchhandlung.

6 La traducción inglesa de las referencias de Hoffmann relativas a Mendel puede consultarse en OlBy, R. \& GAUTREY, P. (1968), pp. 9-10; OLBY, R. (1985), p. 222.

7 La conexión fue establecida por PUNNET, R. C. (1925), «An early reference to Mendel's work», Nature, 116, 606. También OLBY, R.; GAUTREY, P. (1968); y OLBY, R. (1985), appendix to chapter 5. 
dejó pasar esta oportunidad ${ }^{8}$. En este período investigaba sobre la fecundación vegetal -The effects of cross and selffertilisation in the vegetable kingdom fue el resultado de once años de experiencias ${ }^{9}-$, y hubiera podido discutir con conocimiento de causa la tesis mendeliana sobre la transmisión de caracteres en la reproducción sexual ${ }^{10}$.

\section{GUISANTES MÁGICOS}

Pisum sativum fue una de las especies elegidas por prestigiosos horticultores como Thomas Andrew Knight, John Goss, Alexander Seton y Carl Friedrich von Gärtner ${ }^{11}$, para sus experimentos de hibridación. Mendel no fue innovador en su elección. Tampoco lo fueron sus observaciones. La dominancia, la constancia morfológica de la primera generación híbrida, la segregación morfológica de la segunda generación, era una fenomenología ya observada y referida por los citados Knight, Gärtner, Goss, Seton y otros como Maxwel T. Masters, cuyo trabajo conocía Darwin detalladamente ${ }^{12}$, y Josep Kölreuter ${ }^{13}$, citado frecuentemente por Mendel ${ }^{14}$. Cuestión

8 Años más tarde, la lectura del libro de Wilheelm Olbers Focke, Die Pflanzen-Mischlinge: Ein Beitrag zur Biologie der Gewächse, 1881 -obra donde se reseña el trabajo de Mendel y que Darwin recibió inmediatamente tras su publicación-, y su amistad con George John Romanes -autor del artículo «Hybridism» para la novena edición de la Enciclopaedia Britannica (1875-1889), donde también se cita a Mendel-, fueron otras oportunidades para conocerle. Resulta improbable que al final de su vida, falleció en 1882, Darwin no tuviese noticias de su trabajo, pero el hecho es ya poco relevante, incluso anecdótico, serán otros los encargados de incorporar las leyes mendelianas al ideario evolucionista, Hugo de Vries y Thomas H. Morgan principalmente. Además de OlBY, R. y GAUTREY, P. (1968); WeInSTEIN, A. (1977); Olby, R (1985); véase RomAnES, L.G. (1896), The life and letteres of George John Romanes, Londres; Edwardson, J. R. (1962), «Another reference to Mendel before 1900», Journal of heredity, vol. 53, p. 152.

9 Cf. DARwin, F. (ed.) (1958), The autobiography of Charles Darwin and Selected letters, New York, Dover, pp. 51, 330 (1 ${ }^{\mathrm{a}}$ ed.1892). Edición en castellano: DARWIN, F. (ed.) (1997), Charles Darwin, autobiografia y cartas escogidas, Madrid, Alianza.

10 Los principales estudios botánicos de Darwin pertenecen a esta época: Fertilization of orchids, 1861 (1877, 2 $2^{\mathrm{a}}$ edición muy ampliada); The Insectivorous Plants, 1875; The effects of cross and self fertilisation in the vegetable kingdom, 1876; The different Forms of Flowers, 1877 (2 $2^{\mathrm{a}}$ ed. 1880).

11 KNight, A. T. (1799), «An account of some experiments of the fecundity of vegetables», Philosophical Transactions, pp. 195-204; GÄRTNER, C. F. (1849), Versuche und beobachtungen über die Bastarderzeugung im pflanzenreiche, Stuttgart; Goss, J. (1824), «On variation in colour of peas occasionated by cross-pollination», Transactions of the Horticultural Society of London, vol. 5, pp. 234-5; Seton, A. (1824), «On the variation in the colour of peas from cross-impregnation», Transactions of the Horticultural Society of London, vol. 5, p. 236.

12 Masters fue un cultivador de Canterbury y dedicó gran atención al guisante. Publicó sus trabajos sobre hibridación en revistas como Gardener's Chroniche, 1850; Royal Institution Lecture, 1860; Transactions of the Linnean Society, 1861, Natural History Review, 1863, Journal of Hort., 1861; y Darwin leyó sus trabajos. Cf. The Variation of Plants and Animals under Domestication, Londres, 1868, 2 vols. Sobre los precursores de Mendel véase ZIRKLE, C. (1951), «Gregor Mendel \& his Precursors», Isis, vol. 42, pp. 97-104. En este contexto no hay que olvidar las observaciones sobre la segregación de caracteres 
muy diferente es la interpretación que dieron a sus observaciones, nivel de conocimiento donde Mendel fue original. Su análisis estadístico del fenotipo y el subsiguiente desarrollo teórico del genotipo marcó la diferencia frente a sus contemporáneos.

La hibridación, admite Darwin en 1877, era uno de los principales obstáculos para la aceptación y el progreso del principio de la evolución ${ }^{15}$, su interés por el tema no es casual. Pisun sativum fue también un problema para Darwin a causa de la constancia morfológica que mostraban las sucesivas generaciones en sus experiencias de cross fertilisation ${ }^{16}$, circunstancia que no alcanzó a explicar. Su hijo Francis le justifica atribuyendo el resultado a la entomofilia: «El hecho es que no siendo indígena ninguna de las plantas, no están perfectamente adaptadas para la fertilización por los insectos británicos. En esta etapa de sus observaciones no podía saber que la coordinación entre una flor y el insecto particular que la fertiliza puede ser tan delicada como la que existe entre una cerradura y su llave, de modo que difícilmente se le podía ocurrir esta explicación» ${ }^{17}$. Darwin conocía este problema por sus propias investigaciones, y sabía de otras peculiaridades reproductoras del género Pisum por experimentos ajenos. Sabía que dentro de una misma vaina se podían encontrar diferentes tipos de guisantes: redondos y plisados, por ejemplo, y que las plantas provenientes de semillas plisadas tenían una marcada tendencia a producir guisantes redondos ${ }^{18}$. También que obtenidas cuatro subvariedades a partir de una misma planta (azul-redondo, blanco-redondo, azul-plisado, azul-redondo) sembradas por separado durante varios años consecutivos las semillas de cada variedad originaban los tipos correspondientes al conjunto ${ }^{19}$. Que las variedades blanca y azul se cruzan mutuamente cuando están próximas y las vainas contienen en ambos casos semillas de las dos coloraciones, circunstancia habitual al cruzarse variedades de diferente color ${ }^{20}$.

Los guisantes combinaban formas y colores, y lo hacían o por arte de magia o atendiendo a las leyes de la dominancia, la uniformidad, y la segregación de caracteres propuestas por Mendel. No podemos probar que Darwin conociese los resultados

realizadas por Agustin Sageret en su libro Promologie physiologique, París, 1830; cf. Peter Vorzimmer (1963), «Charles Darwin and blending inheritance», Isis, vol.54, nª 177, p. 383.

13 KÖLREUTER, Joseph Gottlieb (1761-1766), Vorläufige Nachricht von einigen das Geschlecht der Pflanzen betreffenden Versuchen und Beobachtungen, Leipzig; (1777), Das entdeckte Geheimnis der Krytogamie, Karlsruhe.

14 Otros autores referidos en su artículo son: Gärtner, William Herbert, Lecoq, y Wichura. $C f$. MENDEL, G. (1984), pp. 39, 48, 56, 82-5, 88-93.

15 DARWIN, Ch. (1877), p. 28.

16 DARwin, F. (ed.) (1958), p. 321; Darwin, Ch. (1868), De la variation des animaux et des plantes sous l'action de la domestication, París, Reinwald, vol. 1, p. 350; Darwin, Ch. (1877), Des effets de la fecondation croisée et de la fécondation directe dans le règne végétal, París, Reinwald, p. 161.

17 DARWIN, F. (ed.) (1997), p. 550-1; Darwin, F. (ed.) (1958), p. 321.

18 DARWIN, Ch. (1868), p. 350.

19 Ibidem.

20 Ibidem, p.421. 
de esta investigación, pero, indudablemente, estos fenómenos pasaron ante sus ojos y su explicación del mecanismo hereditario fue muy diferente a la ofrecida por Mendel.

\section{TEORÍA DE LA PANGÉNESIS}

Cualquier teoría sobre la evolución de las especies terrestres tiene que demostrar dos principios básicos: a) la aparición de la flora y fauna actuales por la transformación de antepasados extintos; b) la existencia de un mecanismo reproductor acorde con el argumento transformista. Darwin publicó en 1859 la primera edición de $O n$ the origin of species pero hasta 1868 no expuso un modelo hereditario complementario a su teoría de la evolución, aunque la idea se remonta a comienzos de la década de los años cuarenta ${ }^{21}$. Lo denominó pangenesis theory y constituye el capítulo final del libro The Variation of Plants and Animals under Domestication ${ }^{22}$, cuya primera edición, 1500 ejemplares, se vendió en una semana ${ }^{23}$. Explicar cómo se producen y se transmiten las variaciones a la progenie en el curso de la especiación fue el reto planteado por El origen de las especies, pero Darwin postergó la resolución del problema convirtiéndolo en un argumento contrario a su ideología. Por ejemplo, para un darwinista como Wallace la carencia de un modelo hereditario complemento a la teoría de la evolución había sido una dificultad inquietante que la pangénesis resolvía ${ }^{24}$.

Darwin eligió la teoría celular como referente científico de su propuesta, y vertebró la pangénesis entorno a dos fenómenos citológicos: 1) la división celular; 2) la producción de partículas celulares, gémulas, que circulan libremente por el organismo y tienen capacidad individual para regenerar la célula de procedencia ${ }^{25}$. Relacionando ambos procesos propone un mecanismo fisiológico común a todos los seres vivos y activo en cualquier fenómeno hereditario: en su fase de división las células producen gémulas que son liberadas al sistema, el conjunto representa físicamente el

$21 C f$. la carta dirigida por Darwin a Lyell en agosto de 1867, donde afirma que la pangénesis tenía una antigüedad de 26 ó 27 años; rep. en DARWIN, F. (ed.) (1958), p. 281. Sobre este asunto es relevante que en 1860 Darwin iniciase la elaboración de The Variation of Plants and Animals under Domestication y cuatro años después escribía a J. Hooker notificándole que había comenzado a repasar el manuscrito del libro. En 1865 enviaba a T. H. Huxley un manuscrito sobre la pangénesis. Cf. Ibidem, pp. 280-281; HuXLEY, L. (1903) Life and letters of Thomas Henry Huxley, Londres, v. I, p. 387. También OLBY, R.C. (1963), «Charles Darwin's Manuscript of Pangenesis», The British Journal for the History of Science, vol. 1, pp. 251-263; que reproduce el manuscrito.

22 Para este artículo hemos utilizado la traducción francesa incluida en LENAY, Ch. (ed.) (1990), $L a$ découverte des lois de l'hérédité.Une anthologie, Inglaterra, Presses Pocket, pp. 105-161; en adelante Darwin (1990).

23 Carta de Ch. Darwin a J.D. Hooker, 10 de febrero de 1868; rep. en DARWIN, F. (ed.) (1958), p. 282.

24 DARWIN, F. (ed.) (1958), p. 282.

25 DARWIN (1990), p. 125. 
organigrama anatómico individual permaneciendo inactiva su capacidad generatriz si no ocurre algún proceso multiplicativo. Entonces, cada unidad desarrolla una célula semejante a la progenitora, punto de partida para la morfogénesis de nuevos organismos en los procesos reproductivos, o para reconstruir la porción anatómica lesionada si es un episodio regenerativo. Durante la regeneración sólo se activan aquellas gémulas procedentes de la región mutilada, utilizando como patrón de agregación el remanente histológico. Para la reproducción hay un almacenamiento previo de las gémulas en las zonas y órganos reproductores donde se agregan por afinidad mutua $^{26}$. Yemas y gametos son simples contenedores de materia a partir de la cual se genera el nuevo individuo ${ }^{27}$. La fecundación provoca en la reproducción sexual la unión de los gametos mezclándose ambos grupos de gémulas. El agregado resultante combina los caracteres pertenecientes a los progenitores, estableciéndose las nuevas coordenadas anatómicas que regularán la embriogénesis ${ }^{28}$. Las gémulas pueden permanecer latentes durante sucesivas generaciones transmitiéndose a la descendencia mediante la reproducción. Su reactivación fortuita provoca la reaparición de caracteres ancestrales en la serie genealógica ${ }^{29}$. Con este esquema conceptual se establecen dos niveles hereditarios complementarios al separar la manifestación tipológica de un caracter y su transmisión individual ${ }^{30}$, en correspondencia con los futuros conceptos de fenotipo y genotipo

Al elaborar su pangenesis theory Darwin siguió la pauta del materialismo biológico $^{31}$ impulsada por la teoría celular. Las gémulas son mínimas porciones de contenido celular ${ }^{32}$ y se comportan como entidades biológicas independientes que tienen su propia función nutricional y se multiplican por división ${ }^{33}$. Representan la mínima porción de materia orgánica con capacidad vital. También tuvo presente el lema Omnis cellula e cellula ${ }^{34}$ acuñado por Rudolf Virchow como símbolo de la continuidad de la materia viva a través de la división celular ${ }^{35}$. Continuidad que la pangénesis reproduce a escala macroscópica pues las gémulas unen físicamente a los padres con sus hijos. El fenómeno de la herencia es la consecuencia de un mecanismo fisiológico

26 Ibidem, pp. 125, 128.

27 Ibidem, pp. 106, 125, 129.

28 Ibidem, pp. 132, 136-7. En el caso de la reproducción sexual Darwin plantea la posibilidad de una agregación especial, en pequeña cantidad, de las gémulas. Ambos gametos, o sólo el femenino, incluirían gémulas exclusivas provinientes de células primordiales que regulan el desarrollo. $C f$. pp. 129, 144.

29 Ibidem, pp. 124, 156-7.

30 Ibidem, p. 123.

31 Cf. la carta de Darwin a J. Hooker, febrero de 1868. Rep. en DARWIN, F. (1958), p. 282.

32 DARWIN (1990), p. 128.

33 Ibidem, pp. 125, 129, 154, 158

34 DARWIN (1990), p. 120.

35 Virchow Rudolf (1858), Die Cellularpathologie in ihrer Begründung auf physiologischer und pathologischer Gewebelehre, Berlín. 
dinámico, estable y cerrado, por el cual los progenitores donan a los hijos su propia sustancia física. La materia se utiliza como modelo para fotografiar ${ }^{36}$ la morfología de los padres activando las unidades (gémulas) que componen el patrón parental ${ }^{37}$. En consecuencia, la variabilidad tiene un origen externo, proviene de la interacción del individuo con el hábitat. Los cambios en las condiciones de vida repercuten a dos niveles morfológicos: a) influyen sobre los órganos reproductores causando una defectuosa agregación gemular que altera el esquema anatómico embrionario formándose nuevos caracteres; b) actúan sobre el individuo adulto modificando su anatomía. Las células de los órganos afectados emitirán gémulas correspondientes a su nueva estructura por medio de las cuales las modificaciones se incorporan al bagaje hereditario del organismo ${ }^{38}$. Un efecto similar tendría el uso y desuso de un determinado órgano, causando su desarrollo o atrofia ${ }^{39}$. Y no olvida Darwin el factor de variabilidad intrínseco a todo ser vivo por las gémulas en estado latente que porta ${ }^{40}$. El fenómeno justifica la reaparición de caracteres ancestrales pero contraviene el principio de la selección natural, admisible si vinculamos el retorno de esta pretérita morfología a la recuperación de las condiciones de vida precedentes.

La idea darwinista de materializar la herencia de caracteres empleando partículas físicas, en su caso de procedencia celular, era una hipótesis recurrente vinculada al pensamiento transformista. Como admite Darwin, a instancia de Thomas Huxley ${ }^{41}$, una década antes conocidos naturalistas como el conde de Buffon y Charles Bonnet realizaron propuestas semejantes. Contemporáneamente Herbert Spencer surcó el mismo océano de partículas y Darwin era consciente del hecho ${ }^{42}$.

\section{DOMINANCIA Y RECESIVIDAD}

Décadas después de confortarse con la pangénesis Wallace suscribe la teoría del plasma germinal propuesta por Weismann ${ }^{43}$ reconociendo la complejidad y dificul-

\footnotetext{
36 DARWIN, F. (ed.) (1958), p. 281.

37 DARWIN (1990), p. 106

38 Ibidem, pp. 122, 128, 135, 143, 149.

39 Ibidem, pp.150, 153.

40 Ibidem, pp. 124, 151-2

41 Ibidem, p.126-7. Particularmente próximo al esquema darwinista es el modelo de moléculas orgánicas propuesto por Buffon; $c f$. GALERA, A. (1994), «Reflexiones sobre el modelo sistemático, el concepto de especie y el mecanismo de la reproducción en el siglo XVIII», Nouveau monde et renouveau de l'histoire naturelle, París, vol. III, 97-130, pp. 126-130.

42 No olvida Darwin las aportaciones que sobre el tema realizó Richard Owen, Parthenogenesis, 1819; y particularmente Herbert Spencer, Principles of Biology, Londres, 2vols., 1864-7.

43 Entre otras obras $c f$. WEISMANN, A. (1875-6), Studien zur Descendenz theorie, Leipzig, Engelmann, 2 vols. (Studies in the Theory of Descent, Londres, Sampson et al., 1882, incluye un prefacio de
} 
tad de la tesis darwinista, atributos por los que mereció un rechazo generalizado ${ }^{44}$. Su complejidad nace del error conceptual de aplicar el mismo criterio fisiológico para interpretar diferentes procesos biológicos cuyo vínculo es generar materia orgánica. Un argumento repetido, pues también Buffon, por ejemplo, le precedió un siglo en la confusión. Para resolver el problema de la herencia de caracteres Mendel utiliza un método diferente. Ignorando las causas que regulan el fenómeno optó por el experimento y el análisis estadístico de los resultados frente a la especulación. Darwin y é $^{45}$ coincidieron al diagnosticar el problema de la evolución: para comprender la historia evolutiva de las especies terrestres resulta fundamental conocer cómo se transmiten los caracteres en la reproducción sexual.

Mendel eligió el género Pisum por su idoneidad experimental. Las distintas especies ofrecen características constantes y diferentes fácilmente reconocibles, y la particular estructura de sus órganos sexuales dificulta notablemente la contaminación de polen extraño ${ }^{46}$. La solución al problema hereditario planteado la halló observando el número y la variedad de formas producidas en la descendencia híbrida. Cuantificando la aparición de los caracteres seleccionados en los cruzamientos a lo largo de la secuencia generacional calculó su proporción estadística ${ }^{47}$. El resultado son las denominadas leyes mendelianas, tan conocidas que nos ahorraremos el detalle. La identidad morfológica de los individuos que componen la primera generación y la segregación de los caracteres parentales en las siguientes resumen su formulación. De ellas nos interesa resaltar el principio biológico propuesto: las sucesivas generaciones de híbridos manifiestan una tendencia a perpetuar la tipología parental, que se hace mayoritaria frente al tipo híbrido sin llegar a desaparecer ${ }^{48}$. En el marco evolutivo la reaparición de los caracteres parentales significa que la fecundación cruzada no es un eficaz mecanismo de especiación y no puede gobernar la evolución de las especies en los términos planteados por Darwin. Observar que los híbridos repiten las formas parentales no fue original de Mendel, quien conocía las informaciones vertidas al respecto por Gärtner y Kölreuter ${ }^{49}$, pero él demostró experimentalmente el hecho en el caso del guisante y supo extraer su significado hereditario. Llegados a este punto la cuestión es ¿cómo explicar biológicamente este suceso estocástico? Mendel extrapoló los resultados matemáticos al área biológica estableciendo un sencillo mecanis-

Charles Darwin); WeISMAnN, A. (1892), Das Keimplasma. Eine theorie der Vererbung, Jena, G. Fischer (The Germ Plasm. A theorie of Heredity, Londres, H. Ellis \& W. Scott, 1893).

44 Wallace A.R. (1912), Darwinism. An exposition of the theory of natural selection with some of its applications, Londres, Macmillan, p. 438 ( $1^{\mathrm{a}}$ ed. 1889). Sobre la poca aceptación de la pangénesis $c f$. también DARWIN, F. (ed.) (1958), p. 493.

45 Mendel, G. (1984), pp. 39-40.

46 Ibidem, p.41.

47 Ibidem, p.40.

48 Ibidem, p.56.

49 Ibidem. 
mo combinatorio circunscrito a las células germinales, mecanismo regulado por la relación de dominancia que se establece entre caracteres análogos y su segregación al formarse los gametos en los individuos de ambos $\operatorname{sexos}^{50}$. La unión gamética resultante de la fecundación aporta al embrión dos caracteres para cada rasgo tipológico. Cuando los caracteres difieren, híbrido, la forma dominante se manifiesta morfológicamente en detrimento de la recesiva que queda latente, o bien aparece una cualidad intermedia cuando no existe relación de dominancia y los caracteres permanecen ocultos. Esta dualidad factorial convierte la gametogénesis en un proceso fisiológico cuya función es separar la pareja de caracteres reunidos por la fecundación ${ }^{51}$, distribuyéndose individualmente en las células gaméticas que se combinan autónomamente en la reproducción ${ }^{52}$. La expresión tipológica es sólo una parte del potencial morfológico heredado por el individuo, que sólo en los gametos se reparte totalmente. Con este planteamiento Mendel, implícitamente, desarrolla los conceptos de genotipo y fenotipo, y propone una sencilla ley combinatoria como ley general de la herencia ${ }^{53}$. A nivel citológico su propuesta se inserta, también, en el marco de la teoría celular, considerando los gametos como células ${ }^{54}$ y distinguiendo la línea celular germinal de la somática tanto por su exclusiva función hereditaria como por particular fisiología responsable de la segregación factorial de los caracteres.

\section{ESPECIES Y VARIDADES}

En el modelo mendeliano el híbrido representa la variabilidad reproductora, consecuentemente es el origen de la especiación. El fenómeno ocurre cuando el híbrido adquiere estabilidad tipológica y transmite constantes sus caracteres a la descendencia. Cuando sucede aparece una nueva especie ${ }^{55}$. Mendel sustenta esta hipótesis en las observaciones sobre estabilidad híbrida realizadas por Gätner y Wichura en los géneros Aquilegia, Lavatera, Geum, y Dianthus, y atribuye el fenómeno a un proceso fisiológico concordante con su teoría factorial de la herencia. En la fecundación los gametos se unen formando una sola célula. Si las células gaméticas son complementarias la célula resultante es estable, se produce la unión permanente de sus contenidos celulares y el individuo adulto producirá gametos correspondientes sólo al tipo

50 Ibidem, pp.48, 65-71, 86

51 Ibidem, p.87.

52 Ibidem, p.56, 71, 74.

53 Ibidem, p.75.

54 Células germinales, en general, u ováricas y polínicas, en particular es la denominación empleada por Mendel para los gametos, y considera la fecundación como un proceso fisiológico cuyo resultado es la fusión de los gametos formando una nueva célula. $C f$. p. 85-6.

55 Ibidem, p. 85. 
híbrido ${ }^{56}$. Este proceso de fusión supone la reunión de los dos caracteres parentales en una unidad de valor morfológico único que durante la gametogénesis se mantiene constante sin desdoblarse en las unidades originarias. Si los gametos fusionados no se complementan la célula base es inestable y su contenido celular tiene un valor transitorio que permite la disyunción gamética de los caracteres parentales ${ }^{57}$. Al margen de errores, la hipótesis mendeliana tiene el valor teórico de interpretar la especiación como un fenómeno de índole exclusivamente biológica, ajeno a factores externos y restringido a la línea germinal. Es, simplemente, una consecuencia de la reproducción de los organismos.

Según el ideario mendeliano las especies evolucionan pero no influenciadas por el medio $^{58}$ ni dirigidas por la selección natural, el azar es responsable de combinar los caracteres parentales y de fijarlos en los híbridos como especies puras. El fenómeno es discontinuo y la modificación de los caracteres se produce por medios biológicos. Con estos principios Mendel suscribe una corriente evolutiva alternativa al darwinismo vinculada directamente con el neodarwinismo weismanniano y el mutacionismo de Hugo de Vries. Sin duda, William Bateson tenía justificadas razones para afirmar en 1913 que la historia del evolucionismo hubiera sido diferente si Darwin hubiese conocido los experimentos mendelianos ${ }^{59}$. Y tal vez Bateson yerra, pero no al subrayar la importancia del ideario mendeliano sino al suponer que Darwin no conoció sus trabajos.

\footnotetext{
56 Ibidem, pp.86-7.

57 Ibidem. En este punto reconoce Mendel el valor hipotético de su planteamiento por la falta de datos.

58 Ibidem, p. 80.

59 BATESON, W. (1913), Mendel's principles of heredity, Cambridge, University Press, $2^{\mathrm{a}}$ ed., 1913, p. 343 ( $1^{\text {a }}$ ed. 1909$)$.
} 